\title{
Understanding Bowtie Nanoantennas Excited by a Localized Emitter
}

\author{
Victor Pacheco-Peña ${ }^{1}$, Miguel Beruete ${ }^{1,2}$, Antonio I. Fernández-Domínguez ${ }^{3}$, Yu Luo ${ }^{4}$, Miguel Navarro-Cía $^{5}$ \\ ${ }^{1}$ Antennas Group-TERALAB, Universidad Pública de Navarra, 31006 Pamplona, Spain, victor.pacheco@unavarra.es \\ ${ }^{2}$ Institute of Smart Cities, Public University of Navarra, 31006 Pamplona, Spain. miguel.beruete@ unavarra.es \\ ${ }^{3}$ Departamento de Física Teórica de la Materia Condensada and Condensed Matter Physics Center (IFIMAC), Universidad \\ Autónoma de Madrid, Madrid 28049, Spain, a.fernandez-dominguez@uam.es \\ ${ }^{4}$ School of Electrical and Electronic Engineering, Nanyang Technological University, Singapore 639798, Singapore, \\ luoyu@ntu.edu.sg \\ ${ }^{5}$ School of Physics and Astronomy, University of Birmingham, Birmingham B15 2TT, UK, m.navarro-cia@bham.ac.uk
}

\begin{abstract}
A full analytical description of a bowtie nanoantenna excited by a localized emitter is presented using the transformation electromagnetic technique. By applying the conformal mapping, the bowtie nanoantenna is transformed into a periodic multi-parallel plate transmission line problem which can be easily evaluated analytically providing physical insight of the coupling between the dipole nanoemitter and the bowtie nanoantenna. The non-radiative Purcell enhancement spectrum is evaluated both analytically and numerically for different lengths, arm angles and metals, demonstrating a good agreement between both approaches. The method here presented fills the gap of the design techniques for optical nanoantennas.
\end{abstract}

Keywords-conformal transformation, nanoantenna, bowtie nanoantenna, Purcell enhancement.

\section{INTRODUCTION}

Antennas are well-known transducers between guided waves and radiation (and vice versa) for radiofrequency and microwave signals [1]. Recently, antennas have been scaled down to optical wavelengths benefited by the great advance in nanofabrication techniques [2]. These so-called nanoantennas have been proposed for biosensing [3], optical wireless communications [4], nonlinear optics [5], etc. One of the most common nanoantenna geometries is the bowtie due to its high field concentration between the arms over a wide bandwidth. Given the breadth of bowtie nanoantennas, it is necessary to have a method that can provide physical insight about their performance in order to ease their design.

At optical frequencies, the finite conductivity model of metals (used in radiofrequency and microwaves) is no longer valid and dispersive models should be used. This difference hinders the application at optics of the vast knowledge in antenna design from radiofrequency and microwaves. Hence, the design of nanoantennas in general, and bowties in particular, relies strongly up to now on intensive numerical simulations, with few isolated exceptions [6].

Work supported in part by the Spanish Ministerio de Economía y Competitividad [TEC2014-51902-C2-2-R]. V.P.-P. - Spanish Ministerio de Educación, Cultura y Deporte [FPU AP-2012-3796]. M.B.- Spanish Ministerio de Economía y Competitividad [RYC-2011-08221]. Y.L. - NTUA*STAR Silicon Technologies Centre of Excellence [No. 11235150003]. M. N.-C. - University of Birmingham [Birmingham Fellowship].
In this work, the transformation electromagnetic technique is used as an analytical tool to evaluate the electromagnetic response of bowtie nanoantennas [7]. This technique, which allows to work at the level of Maxwell's equations, has been applied before to different nanostructures [8] demonstrating that it can give a full description of the interaction with light and nanoantennas. In this way, the work here presented fills the gap of analytical methodologies for nanoantennas.

\section{AnAlytical Method AND Results}

The sketch of the bowtie nanoantenna is shown in Fig. 1(a). It consists of a nanoantenna with two arms of angle $\theta^{\prime}$, total length $l^{\prime}=L_{1}{ }^{\prime}+L_{2}{ }^{\prime}$ and a gap between the arms illuminated by a line dipole with arbitrary polarization. Here, we will consider that $l^{\prime}<<\lambda_{0}$ to work within the quasi-static approximation where the electric field can be described by an electrostatic potential which satisfies Laplace's equation. This nanoantenna can be transformed to a periodic array of parallel plate transmission lines by applying the conformal mapping $z=$ $\ln \left(z^{\prime}\right)$ to the original coordinates (where $z=x+i y$ and $z^{\prime}=$ $x$ ' $+i y^{\prime}$ are the coordinates of the transformed and the original scenario, respectively). With this transformation the properties of the materials remain the same and the electrostatic potential in both coordinates can be expressed as $\Phi(x, y)=\Phi^{\prime}\left(x^{\prime}, y^{\prime}\right)$ [8].

In the transformed frame (Fig. 1(b)) the dipole triggers surface plasmon polaritons (SPP) into the metal plates and they are reflected back and forward at the end of the slabs. Because of the finite length of the metal slabs, a standing wave pattern is produced generating discrete values of the wave vector $k=$ $(n \pi-\Delta \varphi) /\left(L_{l}+L_{2}\right)$, where $n=1,2 \ldots$ are the discrete SPP modes and $\Delta \varphi$ is the phase correction because of the non-perfect reflection at the end of the slabs, as it will be discussed later.

The process to find the analytical solution is as follows: first the electrostatic potential in each region of Fig. 1(b) is calculated in $k$-space as the sum of discrete transverse modes. Then, boundary conditions are applied. Finally, the induced potential in each region is obtained by applying the inverse Fourier transform to the electrostatic potentials. The electric field in each region can be calculated by simply applying the derivative to each induced potential. 

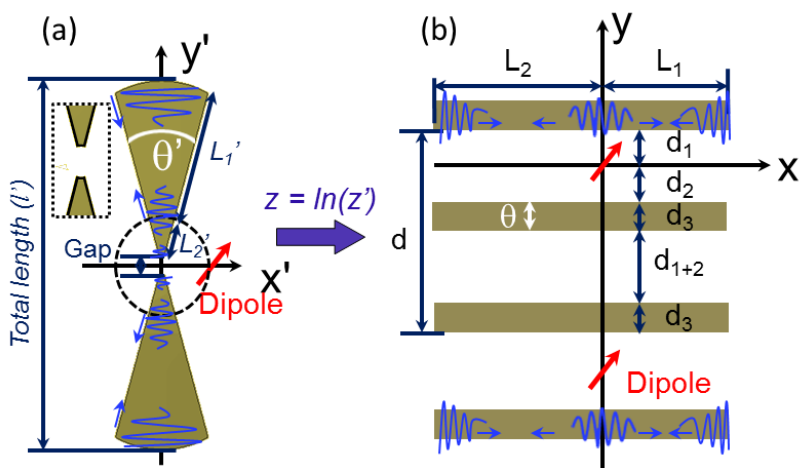

Fig. 1. Bowtie nanoantenna under study (a) and the transformed geometry (b) that consists of a periodic multi parallel plate geometry.

Since the power absorbed in both frames is identical, the non-radiative Purcell enhancement (power absorbed by the bowtie nanoantenna under the dipole illumination with respect to the radiated power by the dipole alone) will be used as a figure of merit to evaluate the performance of the nanoantenna.

We consider a bowtie with a total length $l^{\prime}$, an angle $\theta^{\prime}=$ $20^{\circ}$ for both arms and a gap between the two arms of $1 \mathrm{~nm}$. Gold and silver (with a permittivity fitting Palik's experimental data [9]) are used for the bowtie for comparison purposes. The dipole nanoemitter is placed at $\left(x^{\prime}=1 \mathrm{~nm}, \mathrm{y}^{\prime}=0\right)$. The analytical results of the non-radiative Purcell enhancement spectra for values of $l$ ' ranging from 10 to $20 \mathrm{~nm}$ using a dipole with vertical polarization and the two different metals are shown in Fig. 2(a). Here a correction of phase of $\Delta \varphi=0$ is used in order to discuss the influence of this parameter. It can be observed that high values are obtained (close to $35 \mathrm{~dB}$ ) for all the cases. Two peaks of non-radiative Purcell enhancement are obtained when gold is used while only one is observed for silver within the spectral window shown. This stems from the different dielectric properties of gold and silver.

The numerical values calculated with COMSOL Multiphysics are shown in Fig. 2(b) as continuous lines. By comparing Fig. 2(a,b), one can notice a frequency shift. This is because $\Delta \varphi=0$ refers to the ideal case where the SPPs are perfectly reflected at the end of the parallel plates. However, the SPP has complex (waveguide) impedance, resulting into a complex reflection coefficient. Hence, a phase correction should be applied to account for the complex reflection. The results of $\Delta \varphi$ for each bowtie under study are shown in Fig. 2(c). The longer the bowtie, the smaller the value of $\Delta \varphi$, which holds for both models of metals. This tendency stems from the fact that the SPPs are significantly attenuated at $y^{\prime}{ }_{\text {max }}\left(x_{\max }\right)$ as $l$ ' increases, and thus, the effect of the slab truncation is minimized. After applying the corresponding correction to the analytical model, we obtain the results shown in Fig. 2(b) as symbols. Now it is evident that the analytical and numerical results are the same with both curves overlapped. Moreover, note that with this correction the magnitude of the nonradiative Purcell enhancement is almost $5 \mathrm{~dB}$ above those observed without the phase correction, demonstrating the importance of this parameter on the analytical model. Also, note that the peaks are red-shifted when increasing the length of the nanoantennas as expected in a resonant cavity.

Similar results are derived when looking at a horizontal dipole, but they are not presented here due to page limit.
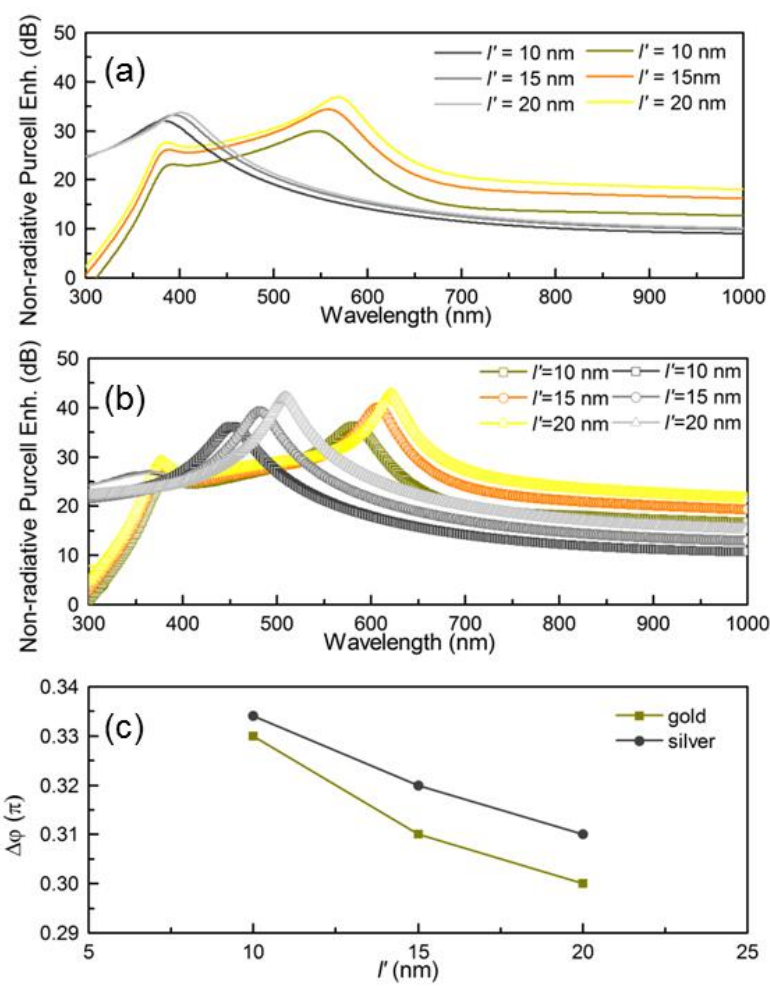

Fig. 2. (a) Analytical non-radiative Purcell spectra without phase correction for several bowtie nanoantennas with different lengths $\left(l^{\prime}\right)$ and angle of the arms $\theta^{\prime}=20^{\circ}$ under vertical polarization of the dipole. (b) Analytical (symbols) and numerical (continuous lines) results of the non-radiative Purcell spectra for the bowtie nanoantennas under study when the phase correction is applied to the analytical model. (c) Phase correction applied.

\section{REFERENCES}

[1] C. A. Balanis, Antenna theory: analysis and design, Third Edit., vol. 72. Hoboken, New Jersey: John Wiley \& Sons, 2005.

[2] P. Bharadwaj, B. Deutsch, and L. Novotny, "Optical Antennas," Adv. Opt. Photonics, vol. 1, p. 438, 2009.

[3] R. Chikkaraddy et al., "Single-molecule strong coupling at room temperature in plasmonic nanocavities," Nature, vol. 535, pp. 1-4, 2016.

[4] D. Dregely et al., "Imaging and steering an optical wireless nanoantenna link," Nat. Commun., vol. 5, no. 4354, 2014.

[5] H. Aouani, M. Rahmani, M. Navarro-Cia, and S. A. Maier, "Thirdharmonic-upconversion enhancement from a single semiconductor nanoparticle coupled to a plasmonic antenna," Nat. Nanotechnol., vol. 9, pp. 290-294, 2014.

[6] C. F. Bohren and D. R. Huffman, Absorption and scattering of light by small particles. Weinheim, Germany: Wiley-VCH Verlag GmbH, 2007.

[7] V. Pacheco-Peña, M. Beruete, A. I. Fernández-Domínguez, Y. Luo, and M. Navarro-Cía, "Description of bow-tie nanoantennas excited by localized emitters using conformal transformation," ACS Photonics, vol. 3, pp. 1223-1232, 2016.

[8] A. Aubry, and J. B. Pendry, "Transformation Optics for Plasmonics," in Active Plasmonics and Tunable Plasmonic Metamaterials, A. Zayats, S. A. Maier, Eds. Hoboken, NJ: John Wiley \& Sons, 2013; pp 105-152.

[9] E. D. Palik, Handbook of Optical Constants of Solids. Academic, 1985 